\title{
Disorder Induced Localized States in Graphene
}

\author{
Vitor M. Pereira, ${ }^{1,2}$ F. Guinea, ${ }^{1,3}$ J. M. B. Lopes dos Santos, ${ }^{2}$ N. M. R. Peres, ${ }^{1,4}$ and A. H. Castro Neto \\ ${ }^{I}$ Department of Physics, Boston University, 590 Commonwealth Avenue, Boston, MA 02215, USA \\ ${ }^{2}$ CFP and Departamento de Física, Faculdade de Ciências Universidade de Porto, 4169-007 Porto, Portugal \\ ${ }^{3}$ Instituto de Ciencia de Materiales de Madrid, CSIC, Cantoblanco E28049 Madrid, Spain \\ ${ }^{4}$ Center of Physics and Departamento de Física, Universidade do Minho, P-4710-057, Braga, Portugal
}

(Dated: August 15, 2018)

\begin{abstract}
We consider the electronic structure near vacancies in the half-filled honeycomb lattice. It is shown that vacancies induce the formation of localized states. When particle-hole symmetry is broken, localized states become resonances close to the Fermi level. We also study the problem of a finite density of vacancies, obtaining the electronic density of states, and discussing the issue of electronic localization in these systems. Our results have also relevance for the problem of disorder in d-wave superconductors.
\end{abstract}

PACS numbers: 81.05.Uw, 71.55.-i, 71.27.+a, 71.23.-k

Introduction. The problem of disorder in systems with Dirac fermions has been studied extensively in the last few years in the context of dirty d-wave superconductors [1]. Dirac fermions are also the elementary excitations of the honeycomb lattice at half-filling, equally known as graphene, which is realized in two-dimensional (2D) Carbon based materials with $\mathrm{sp}^{2}$ bonding. It is well-known that disorder is ubiquitous in graphene and graphite (which is produced by stacking graphene sheets) and its effect on the electronic structure has been studied extensively $[2,3,4,5,6,7,8,9,10,11$, $12,13]$. It has been shown recently [14] that the interplay of disorder and electron-electron interactions is fundamental for the understanding of recent experiments in graphene devices [15]. Furthermore, experiments reveal that ferromagnetism is generated in heavily disordered graphite samples $[16,17,18,19,20]$, but the understanding of the interplay of strong disorder and electron-electron interactions in these systems is still in its infancy. Different mechanisms for ferromagnetism in graphite have been proposed and they are either based on the nucleation of ferromagnetism around extended defects such as edges $[2,8,12,13]$ or due to exchange interactions originating from unscreened Coulomb interactions [21]. Therefore, the understanding of the nature of the electronic states in Dirac fermion systems with strong disorder is of the utmost interest.

In the following, we analyze in detail states near the Fermi energy induced by vacancies in a tight-binding model for the electronic states of graphene planes. We show that single vacancies in a graphene plane generate localized states which are sensitive to the presence of particle-hole symmetry breaking. Moreover, a finite density of such defects leads to strong changes in the local and averaged electronic Density Of States (DOS) with the creation of localized states at the Dirac point.

The model. We consider a single band model described by the Hamiltonian:

$$
\mathcal{H}=-t \sum_{\langle i, j\rangle} c_{i}^{\dagger} c_{j}-t^{\prime} \sum_{\langle\langle i, j\rangle\rangle} c_{i}^{\dagger} c_{j}+\text { h.c. },
$$

where $c_{j}\left(c_{j}^{\dagger}\right)$ annihilates (creates) an electron at site $\mathbf{R}_{j}$ of the honeycomb lattice (the spin quantum numbers are suppressed since we do not consider spin dependent phenomena).
In (1) $t$ is the nearest neighbor hopping energy $(t \approx 2.7 \mathrm{eV}$ in graphene) and $t^{\prime}$ is the next-nearest neighbor hopping energy. In the honeycomb lattice, while $t$ describes the hopping of electrons between the two sub-lattices, $t^{\prime}$ describes the hopping in the same sub-lattice and therefore breaks particle-hole symmetry. The relevance of $t^{\prime}$ for graphene is suggested by different experiments $\left(t^{\prime} \approx 0.2 t\right)$ [14].

Localized states around vacancies. Firstly we consider the particle-hole symmetric case $\left(t^{\prime}=0\right)$, and use the geometry shown in Fig. 1. We analyze a cluster with periodic boundary conditions along the vertical direction, like a zigzag nanotube. In the absence of vacancies, the Hamiltonian can be simplified using the translational symmetry along the vertical direction. The states can be classified by the momentum (in units of $1 / a$, with $a$ the lattice constant) along the vertical axis, $k_{m}=(2 \pi m) / N, m=1, \ldots, N$ where $N$ is the number of unit cells along the vertical axis. The system is metallic if $N=3 M$, where $M$ is an integer. Using this set of momenta, the wavefunction amplitudes in each sublattice $(A, B)$ can be written as $a_{l, j}=\sum_{k_{m}} a_{l, k_{m}} e^{i k_{m} j}$ and $b_{l, j}=\sum_{k_{m}} b_{l, k_{m}} e^{i k_{m} j}$, where $(l, j)$ are the unit cell coordinates in the geometry of Fig. 1. Upon this transformation, the original problem is mapped, for each value of $k_{m}$, into a one-dimensional (1D) tight-binding model along the horizontal direction, with two sites per unit cell. Such 1D problem is characterized by site amplitudes $a_{l} \equiv a_{l, k_{m}}$ and $b_{l} \equiv b_{l, k_{m}}$, and by two effective hoppings, $t$ and $t\left(1+e^{i k_{m}}\right)$; a gauge transformation allows us to make them both real, reading $t$ and $2 t \cos \left(k_{m} / 2\right)$.

For the solution of the impurity problem, it is convenient to define two planes contiguous to the vacancy, as shown in Fig. 1. The two planes belong to the same sub-lattice, opposite to the one where the vacancy resides. A possible localized state at zero energy must: (i) decay as one moves along the horizontal axis away from these planes, (ii) satisfy the bulk tight binding equations arising from the eigenvalue condition $H|\Psi\rangle=E|\Psi\rangle=0$ at these axes and beyond, and (iii) have amplitudes $a_{0, j}^{(L)}$ and $a_{0, j}^{(R)}$ which satisfy the equations,

$$
a_{0, j}^{(L)}=-a_{0, j}^{(R)}-a_{0, j+1}^{(R)},
$$

for all $j$, except at the vacancy $(j=0)$. Having $b_{l, j}^{(L, R)}=0$ 


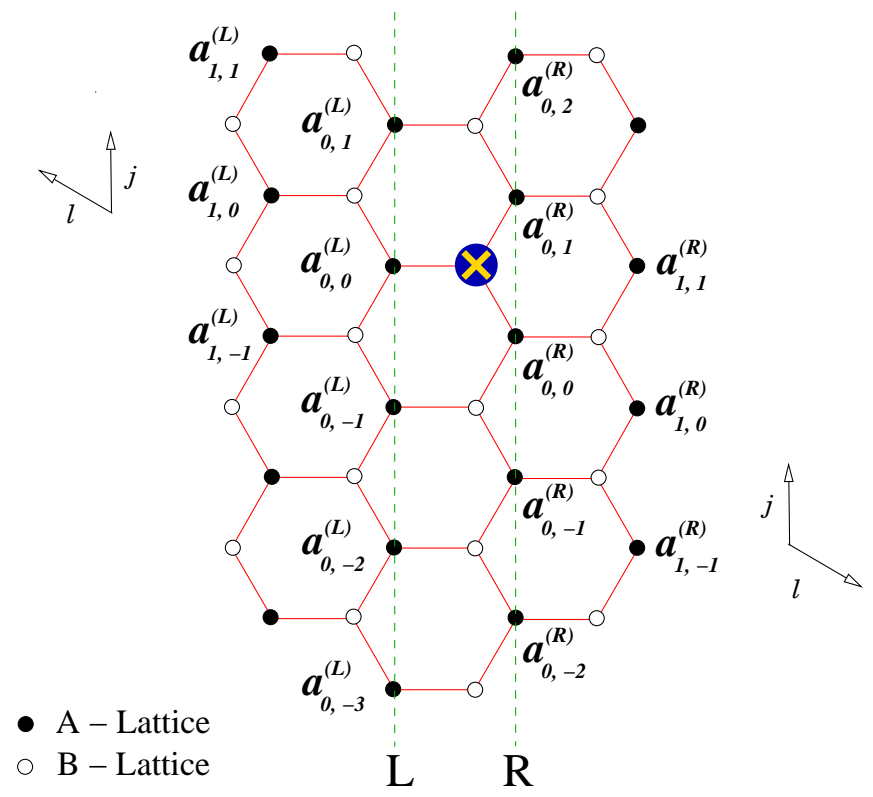

FIG. 1: (color online) Sketch of the geometry considered in the text for the study of a single B-site vacancy $(\otimes)$. The system has periodic boundary conditions along the vertical axis, and is infinite along the horizontal axis. Only some A-site amplitudes are shown.

everywhere automatically satisfies condition (ii). Plane $L$ at the left of the vacancy can be considered a zig-zag edge. To its left, one can define states which decay exponentially for momenta such that $2 \pi / 3 \leq k_{m} \leq 4 \pi / 3[5,6]$. The associated wavefunctions decay as $\left|2 \cos \left(k_{m} / 2\right)\right|^{l}$. As defined in Fig. $1, l$ is always positive, since it grows away from both the $L$ and the $R$ planes, and can be interpreted as the distance to plane $L$ divided by $3 a / 2$. Analogously, one can define localized wavefunctions to the right of plane $R$, for momenta such that $0 \leq k_{m} \leq 2 \pi / 3$ or $4 \pi / 3 \leq k_{m} \leq 2 \pi$. These wavefunctions decay as $\left|2 \cos \left(k_{m} / 2\right)\right|^{-l}$. The amplitudes $a_{0, j}^{(L)}$ and $a_{0, j}^{(R)}$ at planes $L$ and $R$ can be written, in terms of momentum eigenstates, as:

$$
a_{0, j}^{(L)}=\sum_{k_{m}} a_{k_{m}}^{(L)} e^{i k_{m} j}, \quad a_{0, j}^{(R)}=\sum_{k_{m^{\prime}}} a_{k_{m^{\prime}}}^{(R)} e^{i k_{m^{\prime}} j}
$$

and if there was no impurity present in the system, we would

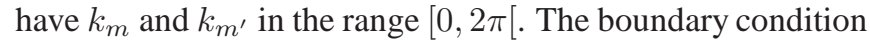
introduced by Eq. (2) can be written as:

$$
\sum_{k_{m}} a_{k_{m}}^{(L)} e^{i k_{m} j}=-\sum_{k_{m^{\prime}}}\left(1+e^{i k_{m^{\prime}}}\right) a_{k_{m^{\prime}}}^{(R)} e^{i k_{m^{\prime}} j}
$$

for $j \neq 0$. These equations admit the solution:

$$
a_{k_{m}}^{(L)}=1, \quad a_{k_{m^{\prime}}}^{(R)}\left(1+e^{i k_{m^{\prime}}}\right)=1,
$$

where the wavevectors $k_{m}$ and $k_{m^{\prime}}$ satisfy $2 \pi / 3 \leq k_{m} \leq$ $4 \pi / 3$ and $0 \leq k_{m^{\prime}} \leq 2 \pi / 3$ or $4 \pi / 3 \leq k_{m^{\prime}} \leq 2 \pi$. We note that these momentum values are the same defining the decaying states to the left and to the right of planes $L$ and $R$, respectively, if we had considered the two cases as separate problems. Hence, we can use the amplitudes we found in the latter case (given above) when constructing the wave function for an impurity. To the left of plane $L$, the amplitude $a_{l, j}^{(L)}$ is now given as $a_{l, j}^{(L)}=\sum_{k_{m}}[-2 \cos (k / 2)]^{l} \exp \left[i k_{m}(j+l / 2)\right]$, where the values of $k_{m}$ are those imposed by the boundary condition, and a similar expression for $a_{l, j}^{(R)}$. For sufficiently wide nanotubes (corresponding to the solution for the infinite lattice) we can approximate the sums in $k_{m}$ by integrals. Shifting from lattice position coordinates to distances relative to the $L$ plane, the amplitude $a_{l, j}^{(L)}$ gives the wavefunction $\Psi(x=l 3 a / 2, y=a \sqrt{3}(j+l / 2))$ at a point of coordinates $(x, y)$. In units of the lattice constant, $\Psi(x, y)$ is approximately

$$
\begin{aligned}
\Psi^{(L)}(x, y) & \sim \int_{2 \pi / 3}^{4 \pi / 3} d k(-2 \cos (k / 2))^{2 x / 3} e^{i k y / \sqrt{3}} \\
& \approx \frac{e^{(4 \pi i y) /(3 \sqrt{3})}}{x+i y}+\frac{e^{2 \pi i(x+y / \sqrt{3}) / 3}}{x-i y}
\end{aligned}
$$

when the lattice site $(x, y)$ is in the opposite sub-lattice of the vacancy, and $\Psi(x, y)=0$ when $(x, y)$ is in the same sublattice as the vacancy. It is now clear that extra vacancies added to the sub-lattice where the impurity resides, cannot change this wave function.

We would like to understand this results, from the point of view of a low-energy effective theory. The eigenstates of the discrete Hamiltonian (1) can be approximated, at long wavelengths, by the Dirac equation. The wavefunctions can be written as a spinor:

$$
\Psi(x, y) \equiv\left(\begin{array}{c}
\psi_{1}^{a, b}(x, y) \\
\psi_{2}^{a, b}(x, y)
\end{array}\right)
$$

where the functions $\psi_{1}(x, y)$ and $\psi_{2}(x, y)$ correspond to the amplitudes of the wavefunctions in each of the two sublattices. There are two set of spinors, $a, b$, which correspond to the two inequivalent states at the corners of the Brillouin zone. At zero energy, the functions at one of the Dirac points satisfy: $\partial_{z} \psi_{1}^{a}(z, \bar{z})=0, \partial_{\bar{z}} \psi_{2}^{a}(z, \bar{z})=0$, where $z=x+i y$ and $\bar{z}=x-i y$; and those at the other Dirac point can be obtained by replacing $z$ by $\bar{z}$ everywhere. The result of Eq. (6) implies that the boundary conditions at the vacancy are such that the combination

$$
\Psi(x, y)=\left(\begin{array}{c}
\psi_{1}^{a}(z)+\psi_{1}^{b}(\bar{z}) \\
0
\end{array}\right) \propto\left(\begin{array}{c}
\frac{1}{z}+\frac{1}{\bar{z}} \\
0
\end{array}\right)
$$

is selected (notice that, in the long wavelength limit, the phase factors in Eq. (6) are factored out upon defining the slowly varying Dirac fields). This solution, although decaying away from the impurity, is not normalizable. It has the same spatial dependence as the quasi-localized solutions which are induced by radial potentials on 2D Dirac fermions [22]. The matching of localized states described above cannot be generalized to the case $t^{\prime} \neq 0$, as the band of edge states is not degenerate in energy [23]. The localized state at the Fermi level 


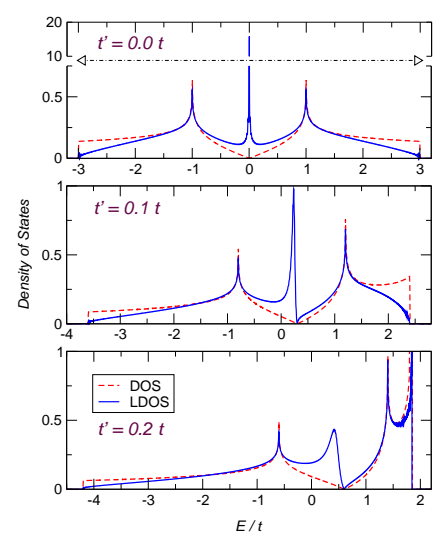

(a)
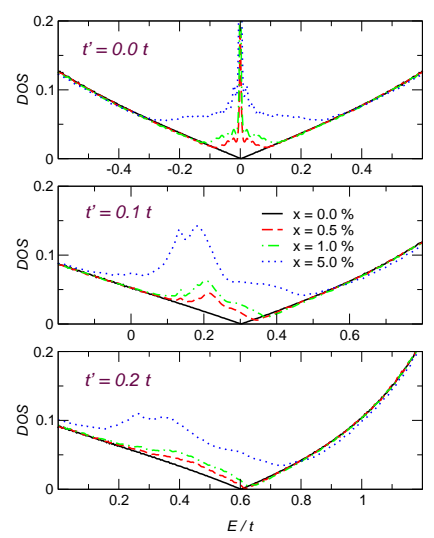

(b)
FIG. 2: (color online) (a) Comparison between the local DOS in the vicinity of a vacancy (blue/continuous) with the bulk DOS (red/dashed) in clean systems. (b) Total DOS in the vicinity of the Dirac points for clusters with $4 \times 10^{6}$ sites, at selected vacancy concentrations. Numerical results in $(\mathrm{a}, \mathrm{b})$ obtained for $t^{\prime}=0$ (top panels), $t^{\prime}=0.1 t$ (center) and $t^{\prime}=0.2 t$ (bottom). (Notice the scale truncation in the upper part of the first panel in (a).

becomes a resonance inside the continuum of extended states. Numerical results for the local (DOS) at a site near a single vacancy are shown in Fig. 2(a). In the absence of electronhole symmetry, the localized state becomes a resonance with increasing width, shifted from the Fermi energy.

The scheme used here can be generalized to study other lattice representations of the Dirac equation with electronhole symmetry. The electronic structure near impurities in d-wave superconductors has been studied using the Hamiltonian: $[24,25,26]$ :

$$
\mathcal{H}_{\mathrm{BCS}}=t \sum_{\langle i, j\rangle} c_{i}^{\dagger} c_{j}+\Delta\left(c_{i, j}^{\dagger} c_{i \pm \mathbf{a}, j}^{\dagger}-c_{i, j}^{\dagger} c_{i . j \pm \mathbf{b}}^{\dagger}\right)+\text { h.c. }
$$

where the sites are defined in a square lattice, $\mathbf{a}$ and $\mathbf{b}$ are the lattice vectors along the horizontal and vertical directions. The Hamiltonian (9) is formally identical to a tight-binding model on a square lattice and two orbitals per site. The hopping terms between different orbitals have different signs along the horizontal and vertical axis. If periodic boundary conditions are applied along the $(1,1)$ and $(1,-1)$ directions, the problem can be written as the matching of a set of 1D wavefunctions, in a way similar to the scheme depicted in Fig. 1. A vacancy leads to a quasi-localized state, described by the same long wavelength wavefunction (see also [27]). The quasi-localized state described here, being localized in a single sub-lattice, remains a solution when there are two vacancies in different sub-lattices, in agreement with numerical calculations [28].

Finite densities of vacancies. We have extended the previous results to systems with a finite density of vacancies, using the stochastic recursion method to obtain the DOS in clusters with up to $\sim 10^{6}$ sites. Results for different impurity concentrations and different values of $t^{\prime}$ are shown in Fig. 2(b). In the presence of electron-hole symmetry $\left(t^{\prime}=0\right)$, the inclusion of vacancies brings an increase of spectral weight to the surroundings of the Dirac point, leading to a DOS whose behavior for $E \approx 0$ mostly resembles the results obtained within a Coherent Potential Approximation (CPA) [14]. The most important feature, however, is the emergence of a sharp peak at the Fermi level, superimposed upon the flat portion of the DOS (apart from the peak, the DOS flattens out in this neighborhood as $x$ is increased past the $5 \%$ shown here). The breaking of the particle-hole symmetry by a finite $t^{\prime}$ results in the broadening of the peak at the Fermi energy, and the displacement of its position by an amount of the order of $t^{\prime}$. All these effects take place close to the the Fermi energy. At higher energies, the only deviations from the DOS of a clean system are the softening of the van Hove singularities and the development of Lifshitz tails (not-shown) at the band edge, both induced by the increasing disorder caused by the random dilution. The onset of this high energy regime, where the profile of the DOS is essentially unperturbed by the presence of vacancies, is determined by $\epsilon \approx v_{\mathrm{F}} / l, l \sim n_{\mathrm{imp}}^{-1 / 2}$ being the average distance between impurities.

To address the degree of localization for the states near the Fermi level, the Inverse Participation Ratio (IPR) was calculated via exact diagonalization on smaller systems. For an eigenstate $m$, the IPR is the quantity defined as: $\mathcal{P}_{m}=$ $\sum_{i}^{N}\left|\Psi_{m}(i)\right|^{4}$, the index $i$ labeling the lattice sites. The wavefunction of an extended state has an amplitude equally significant throughout the entire system $\left(\Psi_{m}(i) \sim N^{-1 / 2}\right)$, whence we naturally expect $\mathcal{P}_{m} \sim N^{-1}$. For a localized state, in opposition, its very definition entails the fact that only a finite number of lattice sites will contribute to the normalization, resulting in much higher values of $\mathcal{P}_{m}$. Results for different values of $t^{\prime}$ are shown in Fig. 3 for random dilution at $0.5 \%$. One observes, first, that $\mathcal{P}_{m} \sim 3 / N$ for all energies but the Fermi level neighborhood, as expected for states extended up to the length scale of the system sizes used in the numerics. Secondly, the IPR becomes significant exactly in the same energy range where the DOS exhibits the vacancy-induced anomalies discussed above. Clearly, the farther the system is driven from the particle-hole symmetric case, the weaker the localization effect, as illustrated by the results obtained with $t^{\prime}=0.2 t$. To this respect, it is worth mentioning that the magnitude of the strongest peaks in $\mathcal{P}_{m}$ at $t^{\prime}=0$ and $t^{\prime}=0.1 t$ is equal to the magnitude of the IPR calculated for a single impurity problem [29]. Such results indicate the existence of quasilocalized states at the center of the resonance, induced by the presence of the vacancies. For higher doping strengths, the enhancement of $\mathcal{P}_{m}$ is weaker in the regions where the DOS becomes flat, in agreement with the reasonably good description obtained with the CPA.

Conclusions. We have studied the local DOS near vacancies in graphene planes. The global and local DOS, are valuable tools in the connection between a microscopic theory and the interpretation of local spectroscopic experiments, of which recent scanning tunneling spectroscopy measurements are an example [20]. In agreement with general arguments valid for 


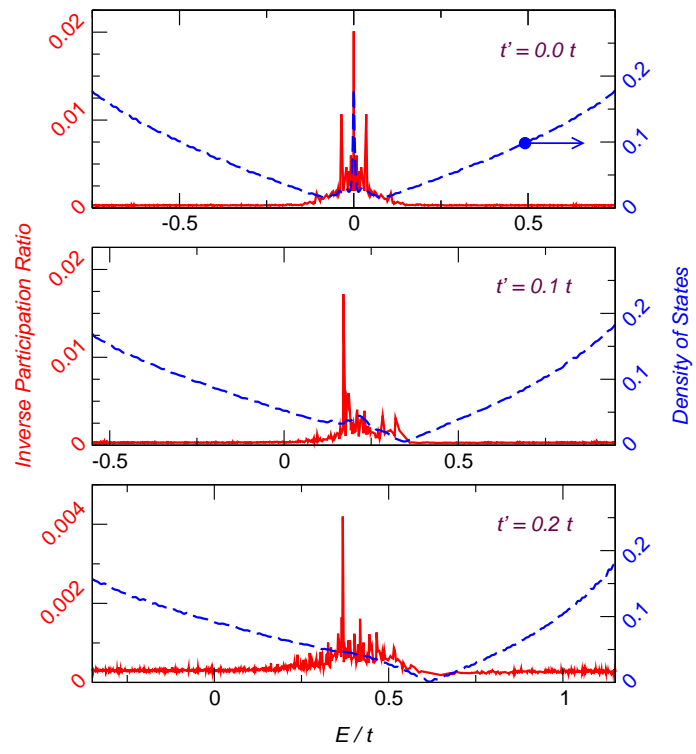

FIG. 3: (color online) Inverse participation ratio for a concentration of vacancies of $0.5 \%$ in systems with $10^{4}$ sites. The dashed curve is the total DOS for the same dilution. Top: $t^{\prime}=0$; center: $t^{\prime}=0.1 t$; bottom: $t^{\prime}=0.2 t$. Only the low energy region is shown.

the 2D Dirac equation, we find quasi-localized states at the Fermi level, if the clean electronic structure shows electronhole symmetry. In the absence of electron-hole symmetry, these states are shifted and broadened. In a system with a finite concentration of impurities, we find a sharp peak built up from localized states, superimposed on a structureless finite DOS, also induced by the vacancies. We have not considered here additional potentials or local atomic displacements which can take place near vacancies. Our findings indicate that the main features in the electronic structure are extended over many lattice sites around the impurity. Thus, we expect that they will be weakly affected by local modifications of the potential near the position of the vacancy. Finally, we have not discussed the implications of our results for the magnetic properties of graphene planes. An enhancement of the density of states over distances which are large compared to the lattice spacing, implies that vacancies may lead to the formation of extended magnetic moments, enhancing the tendency of the system towards ferro or antiferromagnetism.

\section{Acknowledgments}

We thank C. Mudry for valuable discussions. J.M.B.L.S., N.M.R.P. and F.G. are thankful to the Quantum Condensed Matter Visitor's Program at Boston University. V.M.P. acknowledges the support of FCT, through grant SFRH/BD/4655/2001, and Boston University for the hospitality. A.H.C.N. was partially supported through NSF grant DMR-0343790. N.M.R.P. thanks FCT for partially supporting his sabbatical leave. J.M.B.L.S. and V.M.P. were additionally financed by FCT and EU through POCTI (QCAIII).
[1] P. J. Hirschfeld and W. A. Atkinson, J. Low Temp. Phys. 126, 881 (2002).

[2] A. A. Ovchinnikov and I. L. Shamovsky, Journ. of Mol. Struc. (Theochem) 251, 133 (1991).

[3] J. González et al., Phys. Rev. Lett. 69, 172 (1992).

[4] J. C. Charlier et al., Phys. Rev. B 53, 11108 (1996).

[5] K. Wakabayashi and M. Sigrist, Phys. Rev. Lett. 84, 3390 (2000).

[6] K. Wakabayashi, Phys. Rev. B 64, 125428 (2001).

[7] J. González et al., Phys. Rev. B 63, 134421 (2001).

[8] K. Harigaya, Journ. of Phys. C: Condens. Matt. 13, 1295 (2001).

[9] H. Matsumura and T. Ando, J. Phys. Soc. Japan 70, 2657 (2001).

[10] K. Harigaya et al., J. Phys. Chem. Sol. 65, 123 (2004).

[11] E. J. Duplock et al., Phys. Rev. Lett. 92, 225502 (2004).

[12] P. O. Lehtinen et al., Phys. Rev. Lett. 93, 187202 (2004).

[13] M. A. H. Vozmediano et al. (2005), cond-mat/0505557.

[14] N. M. R. Peres et al. (2005), cond-mat/0506709.

[15] K. S. Novoselov et al., Science 306, 666 (2004).
[16] P. Esquinazi et al., Phys. Rev. B 66, 024429 (2002).

[17] P. Esquinazi et al., Phys. Rev. Lett. 91, 227201 (2003).

[18] A. V. Rode et al., Phys. Rev. B 70, 054407 (2004).

[19] T. Makarova and F. Palacio, eds., Carbon-Based Magnetism: an overview of metal free carbon-based compounds and materials (Elsevier, Amsterdam, 2005).

[20] T. Matsui et al., Phys. Rev. Lett. 94, 226403 (2005).

[21] N. M. R. Peres et al. (2005), cond-mat/0507061.

[22] S.-H. Dong et al., Phys. Rev. A 58, 2160 (1998).

[23] P. W. Brouwer et al., Phys. Rev. B 66, 14204 (2002).

[24] W. A. Atkinson et al., Phys. Rev. Lett. 85, 3926 (2000).

[25] W. A. Atkinson et al., Phys. Rev. Lett. 85, 3922 (2000).

[26] W. A. Atkinson et al., Phys. Rev. B 68, 054501 (2003).

[27] G. Khaliullin et al., Phys. Rev. B 56, 11882 (1997).

[28] L. Zhu et al., Phys. Rev. B 67, 094508 (2003).

[29] For a single impurity, $\mathcal{P}_{E=0}$ is seen to vary with $N$ like $\log ^{-2}(N)$, as expected for such quasi-localized state whose normalization depends upon the system size (cfr. Eqs. 6, 8). 